Original article

\title{
Etiological role of opportunistic microflora in the pathogenesis of implant-associated inflammation after primary total knee replacement
}

\author{
Irina V. Babushkina ${ }^{1}$, Alexander S. Bondarenko ${ }^{1}$, Vladimir Yu. Ulyanov ${ }^{1}$, Yulia A. Chibrikova ${ }^{1}$, \\ Ramin G. Adilov' ${ }^{1}$ Ekaterina S. Kupina ${ }^{1}$
}

${ }^{1}$ Saratov State Medical University

Received 28 December 2017, Accepted 26 May 202

Original Text (C) Babushkina I.V., Bondarenko A.S., Ulyanov V.Yu., Chibrikova Yu.A., Adilov R.G., Kupina E.S., 2018, published in Saratov Journal of Medical Scientific Research 2018; 14 (1): 30-34.

(C) 2021, Babushkina I.V., Bondarenko A.S., Ulyanov V.Yu., Chibrikova Yu.A., Adilov R.G., Kupina E.S.

(C) 2021, Saratov Medical Journal

\section{Abstract:}

Objective: to investigate etiological role of opportunistic microflora in the pathogenesis of implant-associated inflammation in patients after primary knee replacement and antibiotic resistance of major infectious agent groups.

Material and Methods. We conducted the retrospective analysis of microbiological samples from 383 patients with postoperative implant-associated inflammation after the primary total knee arthroplasty.

Results. Gram-positive cocci (63.5\%) are the leading agents of implant-associated inflammation. We also noted an increase in the etiological role of coagulase-positive staphylococci. Among the Gram-negative group, Enterobacteriaceae and nonfermenting bacteria were major agents. We revealed high resistance of Staphylococcus spp. to antibiotics of various groups, among which oxazolidinones and glycylcyclines were the most active. Gram-negative bacteria exhibited high resistance to cephalosporine and macrolides, whereas carbapenems and fluoroquinolones worked best.

Conclusion. Gram-negative Enterobacteriaceae, non-fermenters, and Gram-positive cocci are the most significant etiological factors in the pathogenesis of implant-associated inflammation in patients after primary total knee arthroplasty.

Keywords: implant-associated inflammation, etiology, opportunistic microflora, pathogenesis, primary replacement surgery, knee joint.

Cite as Babushkina IV, Bondarenko AS, Ulyanov VYu, Chibrikova YuA, Adilov RG, Kupina ES. Etiological role of opportunistic microflora in the pathogenesis of implant-associated inflammation after primary total knee replacement. Saratov Medical Journal 2021; 2 (2): e0203

Correspondence to Irina V. Babushkina, Tel. +79272233881. E-mail: 10051968@mail.ru

\section{Introduction}

Implant-associated inflammation remains one of the main causes of unsatisfactory outcomes in primary knee arthroplasty [1], which is associated with pathogenetic peculiarities of this typical process, characterized by formation of a microbial biofilm protecting infectious agents due to the polysaccharide complex formation from the effects of nonspecific and specific immunity, as well as from antibacterial pharmaceutical drugs $[1,2]$. Differential diagnosis between infectious complications and aseptic loosening is often problematic due to several factors: the difficulties in microbiological verification of the pathological process, the need for bacterial examination of aspirate or intraoperative samples from periprosthetic tissues, and slow growth of the causative agents of implant-associated inflammation [3]. Quite often, infectious complications caused by low-grade pathogens, such as Staphylococcus epidermidis, are mistakenly regarded as aseptic loosening [4, 5].

Gram-positive cocci often cause early postoperative infection [6]. The widespread distribution of methicillinresistant staphylococcus strains (MRS infection) obstructs the curative therapy of periprosthetic infectious (c) 2021, Saratov State Medical University, Saratov, Russia

(c) 2021, LLC Science and Innovations, Saratov, Russia complications due to resistance to $\beta$-lactam antibiotics and other classes of antibacterial drugs. Late infectious complications are caused as frequently by both Gram-positive aerobic cocci and Gram-negative aerobic and anaerobic bacteria. Currently, special attention is paid to the role of microbial associations and polymicrobial etiology of suppuration after arthroplasty [6].

To select an effective antimicrobial therapy, it is important to identify the so-called difficult-to-treat (DTT) pathogens due to the fact that the choice of effective antibacterial preparations against the microbial biofilm is extremely limited $[6,7]$.

Considering the pathogenetic peculiarities of implantassociated inflammation, it is proposed to take into account, along with the minimum inhibitory concentration (MIC), some more significant indicators, such as the minimum biofilm eradication concentration (MBEC) [7].

Our objective was to investigate the etiological role of opportunistic microflora in the pathogenesis of implantassociated inflammation in patients after the primary knee arthroplasty, along with antibiotic sensitivity testing of the major groups of pathogens. 


\section{Materials and methods}

We conducted a retrospective analysis of microbiological studies based on clinical material samples from 383 patients of the Research Institute of Traumatology, Orthopedics and Neurosurgery of Saratov State Medical University treated from 2014 to 2017 for implant-associated inflammation that developed within 1-12 months after the primary knee arthroplasty. The identification of microorganisms was carried out using conventional techniques (the Order of the USSR Ministry of Healthcare No. 535). Clinical material (wound drainage, aspirate, intraoperative samples of periprosthetic tissues) was plated on selective and differential diagnostic nutrient media. We then studied morphological and cultural characteristics of microorganisms, along with their tinctorial properties. Microorganisms were identified using the BD BBL ${ }^{\text {тм }}$ Crystal ${ }^{\text {тм }}$ auto-reader microbiological analyzer (Becton Dickinson, USA), and the panels: Crystal TM Enteric/Nonfermenter ID Kit, Crystal TM Gram-Positive ID Kit (Becton Dickinson, USA). The sensitivity of microorganisms to antibacterial drugs was determined by the disk diffusion method (Guidelines 4.2.1890-04 Testing Microbial Sensitivity to Antibacterial Medicinal Drugs) using Mueller Hinton Agar (Becton Dickinson, USA) and SensiDisc TM antimycobacterial discs (Becton Dickinson, USA). Methicillin resistance was detected using MeReSa Agar Base - MRSA Alert TM Kit (HiMedia, India). Densi-La-Meter (PLIVA-Lachema Diagnostika, Czech Republic) was used for sample preparation.

Statistical data processing was performed using the IBM ( ) SPSS $®$ Statistics 20 software package. The data largely deviated from the law of normal distribution; hence, the relationships between qualitative features were established via identifying the contingency among them, for which purpose the $\chi^{2}$ statistic was determined. The statistical significance was recognized at $\mathrm{p}<0.05$.

\section{Results}

The analysis of the etiological structure of infectious and inflammatory complications after the primary knee arthroplasty demonstrated a sufficient microbial diversity of various taxonomic groups. The total of 695 samples from 383 patients were examined over 2014-2017. We noted that $48.3 \%$ of the studies isolated a monoculture of microorganisms, $16.9 \%$ of them discovered a microbial association, and $34.7 \%$ of the samples did not yield any bacterial growth. As part of microbial associations, S. aureus was predominantly isolated in combination with Gramnegative non-fermenters or yeast-like fungi of the genus Candida.

The structure of sampled microflora was dominated by Gram-positive cocci, which accounted for $63.5 \%$ of cases. Staphylococcus strains accounted for $53.9 \%$ of cases, of which $34.8 \%$ of isolated strains (S. aureus) were coagulase-positive; $19.1 \%$ were $\mathrm{S}$. epidermidis strains. Other microorganisms belonging to Gram-positive cocci (Streptococcus spp., Enterococcus spp.) were less common (9.6\%) (Figure 1).

Staphylococci were found in $53.9 \%$ of patients as independent causative agent of infectious and inflammatory complications; in $\mathbf{1 1 . 3 \%}$ of examined patients as a part of microbial associations; hence, Staphylococcus spp. strains were found in the total of $65.2 \%$ of patients.

An $18.3 \%$ increase in the proportion of S. aureus in the etiological structure of implant-associated inflammation in 2016-2017 versus 2014-2015 ( $\chi 2=7.15 ; \mathrm{df}=2 ; \mathrm{p}<0.05)$ was (c) 2021, Saratov State Medical University, Saratov, Russia established. The number of infectious complications associated with low-virulent coagulase-negative staphylococci in 2016-2017 declined by $21.1 \%$, compared with 2014-2015 $\left(\chi^{2}=11.43 ; \mathrm{df}=2 ; \mathrm{p}<0.05\right)$.

Representatives of Gram-negative flora were found in $33.4 \%$ of cases. Among them, the most frequently detected microorganisms belonged to the family Enterobacteriaceae (17.3\%). High species diversity of Enterobacteriaceae isolated from patients with purulent complications was revealed: over 14 species. The main representatives of enterobacteria were E. coli (12.8\%) and Enterobacter spp. (4.5\%), other representatives (Klebsiella spp., Kluyvera spp., Proteus spp.) were much less common. Non-fermenting Gram-negative bacteria occurred in $16.1 \%$ of cases, among them $P$. aeruginosa strains and Acinetobacter spp. accounted for $12.8 \%$ and $6.7 \%$, correspondingly.

It should be emphasized that the proportion of Enterobacteriaceae among the representatives of Gramnegative flora increased in 2016-2017 by $12.7 \%$ versus 2014 2015 ( $\chi 2=6.19 ; \mathrm{df}=2 ; \mathrm{p}<0.05)$. Particular attention should be paid to Gram-negative non-fermenters of the genus Acinetobacter: although detected in just $6.7 \%$ of cases, all of their isolated strains were multiresistant.

Yeast-like fungi of the genus Candida were isolated sporadically (3.1\%) as an independent causative agent of implant-associated inflammation; they were found in microbial associations in $38.3 \%$ of cases.

We carried out an antibiogram analysis of 161 staphylococcus strains isolated from patients with infectious and inflammatory complications after primary knee arthroplasty. The sensitivity of microorganisms to 17 antibacterial drugs of seven classes was studied (Figure 2).

The frequency of occurrence of S. aureus strains resistant to methicillin (MRSA) and of S. epidermidis strains with similar resistance (MRSE) was $79.8 \%$ and $57.9 \%$, respectively. Thus, methicillin resistance, which determines resistance to all $\beta$-lactam antibiotics, was higher in S. aureus strains versus $S$. epidermidis strains $\left(\chi^{2}=7.382 ; \mathrm{df}=2\right.$; $\mathrm{p}<0.05$ ).

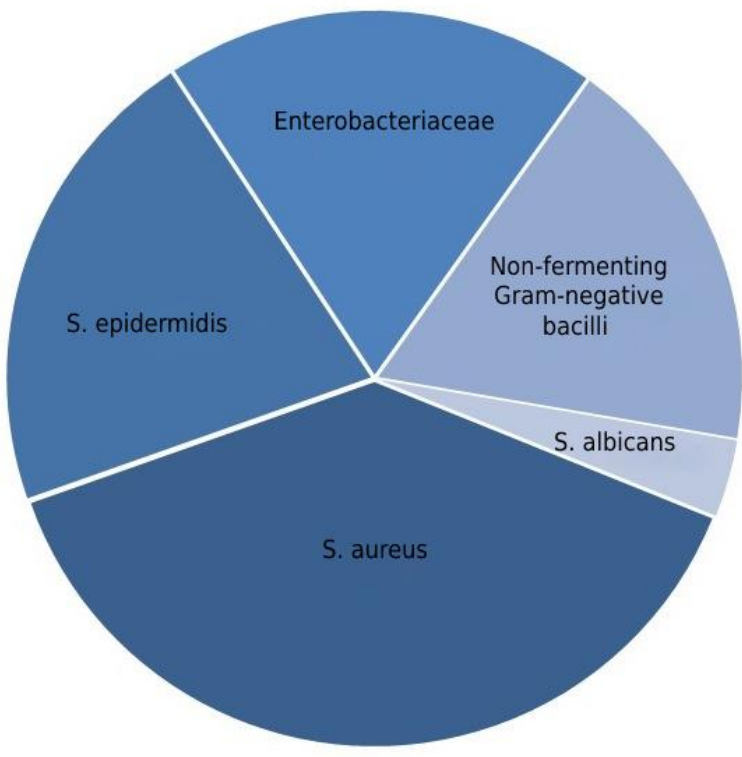

Figure 1. Etiological structure of major causative agents of infectious and inflammatory complications, presented in the form of a monoculture 


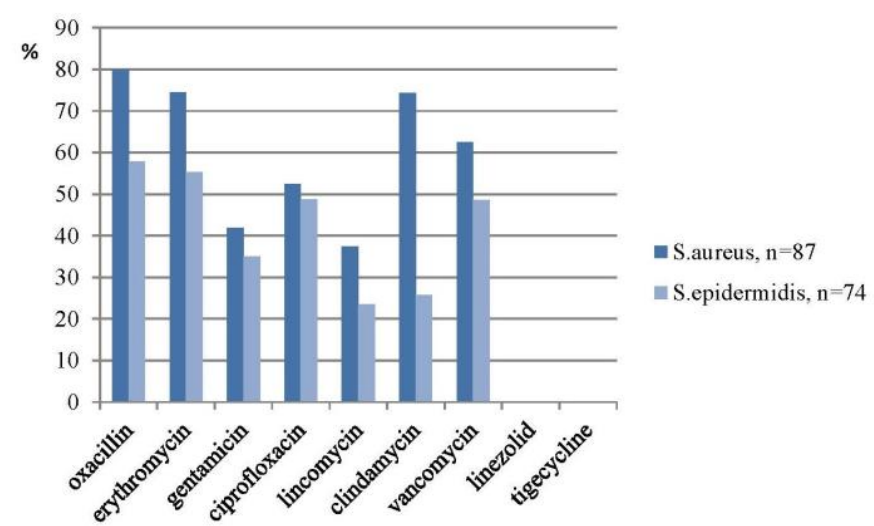

Figure 2. Resistance of Staphylococcus spp. strains to antibacterial medications, \%

The presence of clinical staphylococcus strains resistance to quinolones was revealed. Resistance to ciprofloxacin was detected in $52.4 \%$ of $\mathrm{S}$. aureus strains and $48.7 \%$ of $\mathrm{S}$. epidermidis strains. Resistance to levofloxacin occurred in 48.3 and $27.3 \%$ of strains, respectively. Proportions of S. aureus and S. epidermidis strains resistant to moxifloxacin were $39.8 \%$ and $29.2 \%$, correspondingly. Resistance to erythromycin as a representative of the macrolide group was essential for $74.5 \%$ of S. aureus and $55.2 \%$ of S. epidermidis strains.

We established high level of Staphylococcus spp. resistance to antibacterial drugs of lincosamide family. Clindamycin resistance was observed in $74.3 \%$ and $25.7 \%$ of Staphylococcus aureus and Staphylococcus epidermidis strains, correspondingly, while $37.4 \%$ of S. aureus and $23.5 \%$ of S. epidermidis strains were not sensitive to lincomycin. Lincosamides and glycopeptides are the medicinal drugs of choice for MRS infection, hence the high level of resistance of Staphylococcus spp. to vancomycin: $62.4 \%$ of S. aureus strains and $48.5 \%$ of S. epidermidis strains.

Antibiotics of the oxazolidinone group are prescribed for infectious complications caused by MRS strains with reduced sensitivity to glycopeptides. No resistant strains of staphylococcus were identified for the most widely used antibiotic of this group - linezolid. No resistant strains were also detected for the representative of the glycylcycline class - tigecycline.

Antibiogram analysis yielded the finding that, in the strains isolated from microbial associations vs. pure microbial cultures, the sensitivity to antibacterial drugs was significantly different. In S. aureus pure culture strains, resistance to methicillin determining the resistance to all $\beta$ lactam antibiotics, was found in $79.8 \%$ of cases, while S. aureus strains isolated as part of microbial associations had methicillin resistance in $85.4 \%$ of cases $(\chi 2=9.351 ; p<0.05)$. Among the S. epidermidis pure culture strains, $57.9 \%$ were methicillin-resistant (MRSE); whereas strains isolated from microbial associations had methicillin resistance in $74.9 \%$ of cases $\left(\chi^{2}=7.268 ; \mathrm{p}<0.05\right)$
The isolated clinical strains of Gram-negative bacteria were analyzed for sensitivity to 15 antibacterial drugs, and high strain resistance in Enterobacteriaceae and Gramnegative non-fermenters to conventionally used antibiotics was discovered (Figure 3).

All studied strains of enterobacteria and non-fermenting Gram-negative bacteria were resistant to inhibitor-protected penicillin (amoxiclav). Also, 92.5\% of E. coli strains and $98.7 \%$ of $\mathrm{P}$. aeruginosa strains were found resistant to penicillin of extended spectrum of activity (ampicillin). High resistance level of clinical Gram-negative bacteria strains to cephalosporins was revealed as well: $69.5 \%$ of E. coli strains and $83.0 \%$ of $\mathrm{P}$. aeruginosa strains were resistant to cefazolin. To third-generation cephalosporins (cefotaxime, ceftriaxone, ceftazidime, cefoperazone), resistance was detected in $60.3 \%$ of $\mathrm{E}$. coli strains and $73.1 \%$ of $\mathrm{P}$. aeruginosa strains. Cefepime was not effective for $52.4 \%$ of $\mathrm{E}$. coli strains and $84.2 \%$ of $P$. aeruginosa strains. The resistance of Gram-negative bacteria strains to aminoglycosides was revealed $(62.7 \%$ of $E$. coli strains and $75.1 \%$ of P. aeruginosa strains were resistant to gentamicin, $54.5 \%$ of E. coli strains and $69.3 \%$ of $\mathrm{P}$. aeruginosa strains were not sensitive to amikacin).

The most pronounced activity against strains of Gramnegative bacteria was noted in carbapenems $(75.1 \%$ of E. coli strains and $67.8 \%$ of P. aeruginosa strains were susceptible to imipenem and meropenem). Less obvious activity was observed in antibiotics of the fluoroquinolone group: $63.8 \%$ of E. coli strains and $51.3 \%$ of P. aeruginosa strains were vulnerable to ciprofloxacin, levofloxacin and moxifloxacin.

\section{Discussion}

We established that the leading causative agent of implant-associated inflammation was Gram-positive cocci, represented mainly by $S$. aureus strains and coagulasenegative staphylococci. A tendency towards a further increase in the role of $\mathrm{S}$. aureus in the etiological structure of pathogens in infectious and inflammatory complications after the primary knee arthroplasty was detected.

The widespread distribution of methicillin-resistant strains of S. aureus (MRSA) and S. epidermidis (MRSE) creates a difficult problem in terms of choosing rational antibiotic therapy due to the fact that these strains are resistant to all $\beta$-lactam antibiotics, and also have associated resistance to aminoglycosides, macrolides, lincosamides and tetracyclines.

The substantial role of staphylococci in the etiological structure of orthopedic infection is caused not only by high frequency of their occurrence and high level of acquired resistance to antibacterial preparations of various groups, but also by their ability to quickly form multilevel microbial biofilms on the surface of artificial implants. The presence of pathogens in biofilms complicates diagnosing periprosthetic infection and significantly reduces the effectiveness of antibacterial therapy, including by the drugs that are highly active against MR staphylococci [8]. 


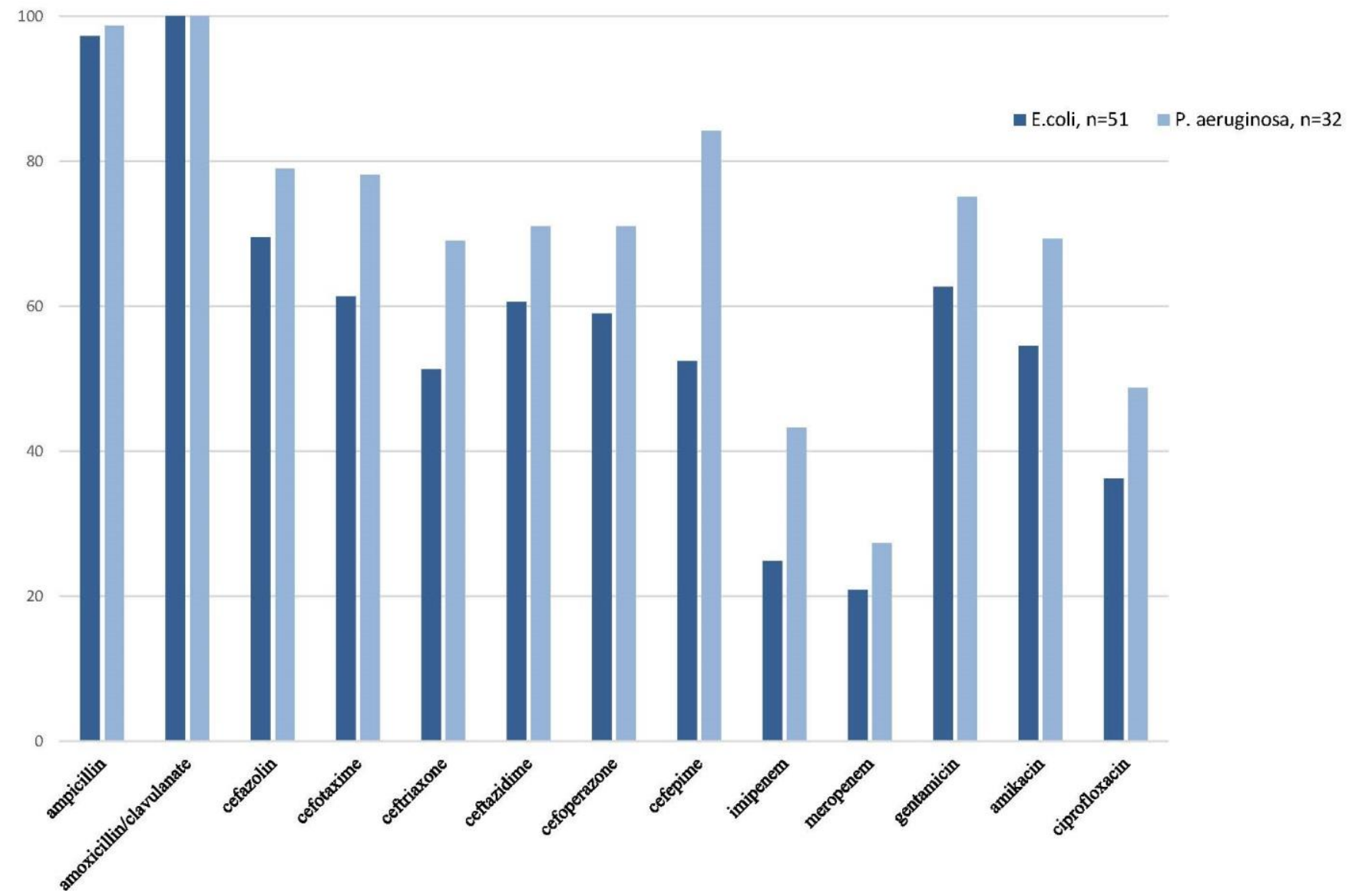

Figure 3. Resistance of clinical strains of gram-negative bacteria to antibacterial medications, \%

Gram-negative bacteria, mainly Enterobacteriaceae, Pseudomonas and Acinetobacter, retain an essential role in the etiological structure of implant-associated inflammation. We discovered high species diversity of Enterobacteriaceae, isolated from patients with purulent complications in 20162017, and noted an increase in their role in the general structure of causative agents of infectious complications after the primary knee arthroplasty. The source of enterobacteria in the hospital is usually the patient per se, so the role of the endogenous route of infection should not be underestimated. The most severe purulent inflammatory complications were caused by non-fermenting Gram-negative bacteria, which occurred in $16.1 \%$ of cases. Their clinical significance was substantiated by multiresistance of the overwhelming majority of strains and a wide range of virulence factors, including their high adhesion capacity leading to the biofilm formation. Nosocomial strains of Acinetobacter spp. often possess antibiotic resistance plasmids and are capable of transferring them to other strains in associates, which seriously complicates effective antibiotic therapy. The presence of microbial associations may indicate an ineffectiveness of a previous antibiotic treatment, which promotes the selection of antibiotic-resistant strains $[9,10]$.

Identification of difficult-to-treat (DTT) pathogens, which include rifampicin-resistant staphylococci, fluoroquinoloneresistant Gram-negative bacteria, and Candida spp., is significant for the choice of treatment tactics and antimicrobial therapy [11].

\section{Conclusion}

The most significant etiological factors in the pathogenesis of implant-associated inflammation in postoperative primary knee arthroplasty patients are Grampositive cocci and Gram-negative representatives of the Enterobacteriaceae family and non-fermenting bacteria.

\section{Conflict of interest}

The authors declare no conflicts of interest pertaining to the study. The study was performed within the framework of the Government procurement No. AAAA-A18-1180202901813 to Research Institute of Traumatology, Orthopedics and Neurosurgery at Saratov State Medical University, Russian Ministry of Healthcare: Optimization of Diagnostic Tactics and Treatment of Latent Periprosthetic Infection in the Knee Joint Area.

\section{References}

1. Klyushin NM, Ababkov YuV, Ermakov AM. Paraprosthetic infection as a cause of revisionary interventions after total knee replacement: Etiology, diagnostics and treatment. The Transbaikalia Medical Bulletin 2015; 2: 189-197. [In Russian].

2. Norkin IA, Shpinyak SP, Girkalo MV, Barabash AP. Outcomes of surgical treatment of infectious complications after large joints arthroplasty. N.N. Priorov Bulletin of Traumatology and Orthopedics 2014; 3: 67-71. [In Russian]. 
3. Bozhkova SA. Current principles of diagnostics and antibacterial therapy of prosthetic joint infection: Review. Traumatology and Orthopedics in Russia 2011; 3: 126-136. [In Russian]

4. Bozhkova SA, Tikhilov RM, Krasnova MV, et al. Orthopedic implant-associated infection: Major infectious agents, local resistance and antimicrobial therapy recommendations. Traumatology and Orthopedics in Russia 2013; 4: 5-15. [In Russian].

5. Masters JP, Smith NA, Foguet P, et al. A systematic review of the evidence for single stage and two stage revision of infected knee replacement. BMC Musculoskeletal Disorders 2013; 14: 222. https://doi.org/10.1186/1471-2474-14-222.

6. Cancienne JM, Granadillo VA, Patel KJ et al. Risk factors for repeat debridement, spacer retention, amputation, arthrodesis, and mortality after removal of an infected total knee arthroplasty with spacer placement. J Arthroplasty 2017; So883-5403 (17): 30760-X. https://doi.org/10.1016/j.arth.2017.08.037.

7. Ulyanov VYu, Luneva IO, Ulyanova EV. Film-forming ability of Staphylococcus aureus nosocomial strains. International Journal of Fundamental and Applied Research 2012; 5: 89. [In Russian].

8. Ulyanov VYu. The film-forming ability of Ps. aeruginosa nosocomial strains. Clinical Microbiology and Antimicrobial Chemotherapy 2012; 14 (2): 52. [In Russian].

9. Babushkina IV, Mamonova IA, Gladkova EV. Etiological role of chronic osteomyelitis agents and effect of copper nanoparticle on clinical Staphylococcus aureus strains. Bulletin of Perm University. Biology 2014; 2: 52-56. [In Russian].

10. Gordinskaya NA, Sabirova EV, Abramova NV et al. Resistance of major infectious agents at divisions of purulent osteology. Traumatology and Orthopedics Issues 2012; 2(1): 14-17. [In Russian).

11. Oliva A, Furustrand TU, Maiolo EM et al. Activities of fosfomycin and rifampin on planktonic and adherent Enterococcus faecalis strains in an experimental foreign-body infection model. Antimicrob Agents Chemother 2014; 58(3): 1284-1293. https://doi.org/10.1128/AAC.02583-12

Authors:

Irina V. Babushkina - PhD, Senior Research Scientist, Division of Fundamental, Clinical and Experimental Studies, Research Institute of Traumatology, Orthopedics and Neurosurgery, Saratov State Medical University, Saratov, Russia;

Alexander S. Bondarenko - Assistant Dean, School of Therapy, School of Clinical Psychology, Saratov State Medical University, Saratov, Russia;

Vladimir Yu. Ulyanov - DSc, Deputy Director for Science and Innovations, Research Institute of Traumatology, Orthopedics and Neurosurgery, Saratov State Medical University, Saratov, Russia;

Yulia A. Chibrikova - Student, School of Therapy, Saratov State Medical University, Saratov, Russia;

Ramin G. Adilov - Student, School of Therapy, Saratov State

Medical University, Saratov, Russia;

Ekaterina S. Kupina - Student, School of Pediatrics, Saratov State Medical University, Saratov, Russia. 